\title{
Le développement d'une langue seconde chez des enfants qui ont un trouble déficitaire d'attention avec hyperactivité (TDAH)
}

\section{S. Frenette, C. Mayer-Crittenden}

Stéphanie Frenette, BScS,

MScS Candidate

Université Laurentienne

sfrenette@laurentian.ca

Chantal Mayer-Crittenden, $\mathrm{PhD}$

Professor, École d'orthophonie

Université Laurentienne

cmayercrittenden@laurentian.ca 


\section{Résumé}

Dans des contextes conversationnels, la pragmatique, qui est l'usage social de la langue, permet de gérer la façon dont les autres aspects du langage sont utilisés (Prutting et Kirchner, 1987; Searle, 1969). Cependant, certains enfants qui ont un trouble déficitaire d'attention avec hyperactivité (TDAH) ou qui ont un trouble du développement du langage (TDL) éprouvent des difficultés avec celle-ci, faisant en sorte qu'ils ont des difficultés à bien communiquer dans des contextes sociaux. Le TDAH est un des troubles psychiatriques les plus communs chez les enfants (Cantwell, 1996). Puisque le TDAH réduit l'habileté à un enfant à porter attention, il y a souvent une interférence avec le processus d'apprentissage. Souvent, il y a plus de difficultés scolaires retrouvées chez les enfants qui ont un TDAH et un TDL (Özerk, Handorff, et Özerk, 2011; Zylowska, Ackerman, Yang, Futrell, Horton, Hale, et coll., 2007).

Le TDL est un trouble caractérisé par des problèmes au niveau de la compréhension et/ou de l'expression du langage sans être causé par un autre trouble ou syndrome. Les orthophonistes sont souvent appelés à évaluer et documenter la coexistence du TDL chez des enfants qui ont un TDAH (American Speech-LanguageHearing Association, 1997). Cependant, évaluer le langage des enfants avec un TDAH ne s'avère pas une tâche facile et comme plusieurs enfants qui ont un TDAH démontrent des difficultés avec l'attention ainsi que l'apprentissage de leur première langue (L1), certains chercheurs stipulent que les problèmes liés au TDAH pourraient également affecter l'apprentissage de la langue seconde (L2).

Toutefois, puisque peu d'étude, sinon aucune, n'ont explorés ce sujet, cette étude aurait comme objectif d'explorer l'impact du TDAH sur l'apprentissage d'une L2. Cette étude exploratoire examinera les habiletés langagières de trois groupes d'enfants, soit des enfants sans TDAH, des enfants qui ont un TDAH et des enfants qui ont un TDAH en plus d'avoir un TDL.

Mots clés : L2, TDAH, TDL, Attention, Apprentissage, Fonctions exécutives, Pragmatique 


\begin{abstract}
In different conversational contexts, pragmatics, which is the social use of language, allows us to manage the way other aspects of language are used (Prutting \& Kirchner, 1987; Searle, 1969). However, some children who have attention deficit hyperactivity disorder (ADHD) or who have a developmental language disorder (DLD) have difficulty with this aspect, making it difficult for them to communicate well in different situations. ADHD is one of the most common psychiatric disorders found in children (Cantwell, 1996). Since ADHD reduces a child's ability to pay attention, these children often have learning difficulties. Often, there are more academic difficulties found in children who have ADHD and DLD (Özerk, Handorff, \& Özerk, 2011; Zylowska, Ackerman, Yang, Futrell, Horton, Hale, \& al., 2007).

DLD is a disorder characterized by problems in understanding and/or expressing language without being caused by another disorder or syndrome. Speechlanguage pathologists are often called upon to evaluate and document the coexistence of DLD in children with ADHD (American SpeechLanguage-Hearing Association, 1997). However, assessing the language of children who have ADHD is not an easy task. As many children with ADHD experience difficulties with attention and learning their first language (L1), some researchers suggests that ADHD may also affect learning of a second language (L2).

However, since few, if any, studies have explored this topic, the aim of this study would be to explore the impact of ADHD on L2 learning. This exploratory study will examine the language skills of three different groups of children: children without ADHD, children with ADHD and children with ADHD and DLD.
\end{abstract}

Key words: L2, ADHA, DLD, Attention, Learning, Executive functions, Pragmatic 


\section{Introduction}

Le développement du langage correspond à un processus complexe et dynamique influencé par plusieurs facteurs comme l'âge de l'enfant, l'exposition à la langue ainsi que les interactions sociales. Un enfant bilingue suit généralement un de deux trajets de l'acquisition du langage, soit le bilinguisme simultané, soit le bilinguisme séquentiel. Pour le bilinguisme simultané, l'enfant acquiert deux langues en même temps avant l'âge de 3 ans tandis que pour le bilinguisme séquentiel, l'enfant acquiert une langue seconde (L2) après avoir acquis sa première langue (Fierro-Cobas et Chan, 2001). À l'âge scolaire, l'acquisition d'une L2 se fait de manière plus intentionnelle contrairement à la petite enfance, soit une période pendant laquelle la langue s'apprend sans que l'enfant s'en rende compte (Fierro-Cobas et Chan, 2001). Parfois, il se peut que des enfants proviennent de foyers où la langue parlée est différente de la langue de scolarisation. Ceci est particulièrement commun dans des communautés linguistiques en situation minoritaire. Ces enfants sont en mesure d'utiliser leurs connaissances et leurs expériences antérieurement acquises dans leur première langue afin de faciliter l'apprentissage de la L2 (FierroCobas et Chan, 2001). Certaines études ont montré que le trouble du développement du langage (TDL) a souvent comme concomitance le trouble déficitaire d'attention avec hyperactivité (TDAH). Ce dernier peut engendrer des difficultés sociales puisque les enfants atteints du TDAH sont souvent de pauvres partenaires conversationnels en raison de leurs déficits en langage pragmatique (Snowling, Bishop, Stothard, Chipchase, et Kaplan, 2006; Tirosh et Cohen, 1998). Puisque très peu d'études ont examiné l'habileté des enfants qui ont un TDAH à apprendre une L2, la présente étude a comme objectif d'explorer cette problématique.

\section{Caractéristiques de l'enfant qui a un TDAH}

Le TDAH est un trouble fréquemment retrouvé chez les enfants. En fait, ce trouble psychiatrique en est un des plus communs chez ceux-ci avec une prévalence de 3 à $10 \%$ de la population d'âge scolaire (APA, 2013; Cantwell, 1996; Hulme et Snowling, 2009; NIH Consensus Development Panel, 2000; LaForette, Murray, et Kollins, 2008; Scahill et Schwab Stone, 2000). Malgré le fait que le TDAH commence à l'enfance, pour plusieurs, il persiste à l'âge adulte. Ce trouble affecte la capacité de l'enfant à contrôler son attention, à s'autoréguler, à planifier, à exécuter une tâche et à surveiller son propre comportement (Barkley, 1997; Hulme et Snowling, 2009). Selon les critères du DSM-V (APA, 2013), le TDAH est caractérisé par une série de comportements qui affectent la performance de la personne atteinte dans un milieu social, éducatif ou vocationnel. Les symptômes sont divisés en deux catégories, soit la catégorie d'inattention, soit celle d'hyperactivité et d'impulsivité. Les symptômes observables de l'inattention incluent : le manque d'attention aux détails ou lors de certaines tâches, l'évitement à entreprendre une tâche qui nécessite un effort mental soutenu ainsi que la distraction. Les symptômes observables de l'hyperactivité comprennent le besoin de continuellement bouger et l'incapacité de rester assis, d'attendre son tour ainsi qu'un discours excessif (APA, 2013; Kim et Kaiser, 2000). Afin d'obtenir le diagnostic du TDAH, les enfants doivent avoir au moins six symptômes parmi les deux 
catégories ou parmi les catégories combinées. De plus, plusieurs des symptômes du TDAH doivent être présents avant l'âge de 12 ans.

$\mathrm{Au}$ cours des dernières années, la recherche sur le TDAH a mené à de nouvelles découvertes en ce qui concerne les troubles concomitants (Barkley, 2006; Douglas, 2005; Guldberg-Kjär et Johansson, 2015; Mayes et Rafalovich, 2007; Nigg, 2005). En fait, il devient de plus en plus évident que certains enfants qui ont un TDAH ont également des troubles de langage importants (Barkley, 2006; Brossard-Racine et coll., 2015; Papaeliou, Maniadaki, et Kakouros, 2015). De plus, les comportements des enfants qui ont un TDAH ont souvent un impact négatif sur leur rendement scolaire. Les élèves ayant des difficultés à se concentrer sur des activités en salle de classe ou à faire leurs devoirs à cause de leurs problèmes d'attention sont susceptibles d'être des apprenants moins efficaces que leurs camarades de classe du même niveau sans trouble d'attention (Gropper et Tannock, 2009). Aussi, les enfants qui ont un TDAH en plus TDL ont plus de difficultés scolaires que ceux qui ont seulement un TDAH (Özerk, Handorff, et Özerk, 2011). De plus, puisque le TDAH réduit l'habileté de porter attention, ce trouble peut causer une interférence avec le processus d'apprentissage, menant à des difficultés scolaires (Canadian Mental Health Association, 2012). En fait, environ $70 \%$ des enfants qui ont un TDAH ont également un trouble d'apprentissage (Mayes, Calhoun, et Crowell, 2000). Un taux élevé d'erreurs sur des tâches jugées comme étant faciles ou l'incapacité à terminer une tâche sont des signes observables en salle de classe qui indiquent qu'il y a probablement une lacune au niveau de l'effort attentionnel (Mayes et coll., 2000).

\section{TDL et TDAH}

Le TDL est un trouble développemental caractérisé par des difficultés avec la compréhension et/ou l'expression du langage qui ne peuvent être expliquées par une autre cause apparente telle qu'une perte d'audition, un retard mental, un trouble émotionnel ou un autre trouble. Autrement dit, c'est lorsqu'un enfant à développement typique a des difficultés langagières dans les sphères réceptives et expressives (Leonard, 1998). Selon Rice (2004), le TDL provoque des difficultés avec l'apprentissage des langues. Ce trouble peut se manifester toute la vie. En fait, Ervin (2001) a montré que $70 \%$ des enfants évalués à 5 ans et qui avaient reçu un diagnostic du TDL continuaient à avoir une performance langagière réduite arrivée à l'âge de 18 à 20 ans. Le TDL se manifeste par une structure du langage (grammaire, morphologie, phonologie) appauvrie, un contenu du langage (vocabulaire) inférieur aux enfants à développement typique (Whitehouse, Line, Watt, et Bishop, 2009) et un usage du langage, soit l'aspect social ou la pragmatique, hors normes (Adams, 2008; Whitehouse et coll., 2009). Ces enfants ont aussi des difficultés en lecture et en écriture (Leonard, 1998). Le TDL affecte environ $7 \%$ de la population générale (Leonard, 1998; Rice, 2004), soit deux enfants par salle de classe (Norbury et coll., 2016). De plus, les enfants qui ont ce trouble vont habituellement apprendre à parler tardivement (Ervin, 2001).

Les orthophonistes sont souvent appelés à évaluer et documenter la coexistence du TDL chez des enfants qui ont un TDAH (American Speech-Language-Hearing Association, 1997). Nous rappelons que le développement du langage chez certains enfants qui ont le TDAH peut être 
compromis (Redmond, Thompson, et Goldstein, 2011). D'ailleurs, le TDAH est le diagnostic psychiatrique le plus fréquemment retrouvé concomitamment chez les enfants qui ont des troubles du langage (Snowling et coll., 2006; Tirosh et Cohen, 1998). Inversement, une proportion significative, soit le deux tiers, d'enfants qui ont un TDAH ont des troubles concomitants du langage (Åsberg, Kopp, Berg-Kelly, et Gillberg, 2010; Bignell et Cain, 2007; Bruce, Thernlund, et Nettelbladt, 2006; Cantwell, 1996; Cohen, Vallance, et Barwick, 2000; Cross, 2011; Helland, Biringer, Helland, et Heimann, 2010; Love et Thompson, 1988; Smalley, 1997; Toplak, Rucklidge, Hetherington, John, et Tannock, 2003). Malgré le fait que ces deux troubles communs coexistent fréquemment, peu d'études comparent directement ces deux populations à incidence élevée (McInnes, Humphries, Hogg-Johnson, et Tannock, 2003; Redmond et coll., 2011). Tannock et Schachar (1996) ont montré dans leur étude épidémiologique que la présence d'un TDL peut coexister chez 35 à $50 \%$ des enfants qui ont des symptômes du TDAH, allant jusqu'à un taux de $90 \%$. Plusieurs études ont montré que, lorsqu'ils sont comparés à des enfants à développement typique, les enfants qui ont un TDAH sont plus à risque de rencontrer des défis dans certains aspects du langage tel qu'un retard des premiers mots et des combinaisons de mots, une pauvre performance sur les tests standardisés (vocabulaire, syntaxe, lecture et la mémoire à court terme), un discours appauvri et des difficultés pragmatiques caractérisées par une participation conversationnelle inappropriée (Barkley, 1997; Cohen et coll., 1988, 1993, 1998, 2000; Love et Thompson, 1988; Purvis et Tannock, 1997; Tirosh et Cohen, 1998). Par ailleurs, le développement neurocognitif des enfants atteint par soit l'un ou l'autre de ces deux troubles ou encore les deux faits en sorte que ces enfants présentent souvent des difficultés avec certaines fonctions exécutives.

\section{Fonctions exécutives, bilinguisme et TDAH}

Les fonctions exécutives, aussi connues sous le nom de contrôle exécutif ou contrôle cognitif, englobent une large gamme de processus mentaux. Plusieurs chercheurs soutiennent qu'il y a trois fonctions exécutives de base, soit l'inhibition, la mémoire de travail et la flexibilité cognitive (Diamond, 2014). Le contrôle d'inhibition est responsable du contrôle de l'attention, du comportement, des pensées et/ou des émotions (Diamond, 2014). Grâce à cette fonction, un individu est en mesure de choisir de porter attention sur un stimulus quelconque et d'ignorer les autres stimuli qui l'entoure, de choisir comment il va se comporter ou réagir plutôt que de se comporter de façon inappropriée ainsi que de contrôler ses propres comportements ou émotions (Diamond, 2014). Cette fonction consiste à résister aux tentations, à ne pas agir de façon impulsive et de pouvoir rester sur une tâche afin de la terminer, malgré les distractions et les tentations d'abandonner (Diamond, 2014). La deuxième fonction exécutive de base, la mémoire de travail, comprend l'habileté à un individu de retenir, manipuler et exploiter l'information momentanément, celle-ci étant fortement liée à la réussite scolaire (Alloway et coll., 2010). Cette dernière est également liée à l'acquisition et à l'exécution de plusieurs compétences cognitives complexes, telles que le langage et la compréhension en lecture (Gathercole, Pickering, Knight, et Stegmann, 2004; Martinussen et coll., 2005; Martinussen et Tannock, 2006). De plus, la mémoire de travail est importante afin de pouvoir mettre un sens à un évènement qui se produit (Diamond, 2014). La 
mémoire de travail peut être divisée en deux catégories, soit la mémoire de travail verbale, soit la mémoire de travail non verbale, aussi connu sous le nom de mémoire de travail visuo-spatiale (Diamond, 2014). La mémoire de travail verbale est utilisée afin de manipuler l'information verbale et joue un rôle important pour tout nouvel apprentissage. Le système de la mémoire de travail verbale a été associé à la capacité d'acquérir de nouvelles connaissances et compétences notamment l'acquisition de la lecture et du langage (Acheson et Macdonald, 2009; Swanson, 2006). Finalement, la flexibilité cognitive, la troisième fonction exécutive de base, permet à un individu de changer de perspective de façon spatiale ou interpersonnelle (Diamond, 2014). Celleci permet également le changement de la façon dont nous pensons, l'ajustement par rapport aux demandes et priorités changeantes ainsi que la capacité à un individu d'admettre qu'il avait tort (Diamond, 2014).

Le bilinguisme est très commun à l'échelle mondiale (Lewis, 2009). Cela est d'autant plus vrai en milieu minoritaire où le bilinguisme s'impose de fait aux minoritaires (Gathercole, 2014). Des études montrent que le fait d'être bilingue peut avoir un impact positif sur le développement des fonctions exécutives (Kovacs et Mehler, 2009; Singh et coll., 2014). Il a été démontré que des enfants et adultes bilingues ont une meilleure performance sur plusieurs tâches de fonctions exécutives par rapport à leurs paires monolingues. Cependant, certaines études n'ont pas observé cette différence. Selon les recherches, la source de cet avantage sur les fonctions exécutives viendrait de l'expérience de vie des bilingues qui utilisent leurs fonctions exécutives pour gérer deux systèmes linguistiques qui, comme il a souvent été montré dans les recherches, sont actifs même lorsqu'une seule langue est utilisée (Bialystok, 2011).

Certaines études qui ont porté sur le TDAH ont montré que ce trouble peut être caractérisé par des difficultés avec certaines fonctions exécutives (Barkley, 2006; Cepeda, Cepeda, et Kramer, 2000; Hervey, Epstein, et Curry, 2004; King, Colla, Brass, Heuser, et Von Cramon, 2007; Kramer, Cepeda, et Cepeda, 2001; Mathers, 2006; Sonuga-Barke, 2003; Sonuga-Barke, Bitsakou, et Thompson, 2010). Malgré le fait qu'il existe plusieurs études sur les troubles de la mémoire de travail chez les enfants qui ont le TDL ou le TDAH (Gathercole et Baddeley, 1990; Gathercole, Brown, et Pickering, 2003; Martinussen, Hayden, Hogg-Johnson, et Tannock, 2005; McGrath et coll., 2011; Rogers, Hwang, Toplak, Weiss, et Tannock, 2011), il existe très peu de recherches sur les effets de la comorbidité du TDL et du TDAH sur la mémoire de travail. À ce jour, il a été démontré que les enfants qui ont un TDL ou un TDAH sont surtout limités quant à leur capacité de mémoire de travail verbal (Alloway, Rajendran, et Archibald, 2009). Cohen et coll. (2000) soutiennent que les troubles de mémoire de travail sont plus fortement reliés au TDL qu'au TDAH chez les enfants âgés de 8 à 12 ans.

Selon une étude qui a inclus des participants bilingues et monolingues ayant un TDAH ayant comme objectif d'explorer l'effet du bilinguisme et du TDAH sur les fonctions exécutives, le bilinguisme et le TDAH pourraient avoir un effet combiné sur les fonctions exécutives. C'està-dire que les désavantages du TDAH sur les fonctions exécutives sont plus prononcés chez les bilingues que chez les monolingues. Cependant, l'étude peut seulement être utilisée comme preuve initiale et a besoin d'investigations futures. 


\section{Pragmatique de l'enfant qui a un TDAH}

Certains chercheurs suggèrent que les enfants ayant un TDAH qui ont des troubles liés aux fonctions exécutives ont aussi des difficultés au niveau de la pragmatique (Tannock et Schachar, 1996; Westby et Cutler, 1994). Afin de pouvoir soutenir une conversation réciproque cohérente, porter attention et se souvenir de ce qui a été dit par le partenaire conversationnel est important. Ces habiletés reposent sur les fonctions exécutives d'attention soutenue et de mémoire de travail (Geurts, Broeders, et Nieuwland, 2010). En plus, il est important que la personne soit en mesure d'inhiber les discussions excessives et d'assurer que le partage d'information soit pertinent, soit des habiletés qui reposent sur les fonctions exécutives de l'inhibition et de la planification de la réponse (Tannock et Schachar, 1996; Westby et Cutler, 1994). De même, les troubles de planification et d'organisation peuvent expliquer les difficultés expressives du langage chez les enfants qui ont un TDAH, comme la difficulté à adapter le discours pour répondre aux besoins d'un auditeur (Purvis et Tannock, 1997). Malgré ces théories sur la relation entre la pragmatique et les fonctions exécutives, il y a peu de recherches sur ces relations en ce qui concerne le TDAH.

Le comportement des enfants qui ont un TDAH peut avoir un effet sur les interactions sociales de ces derniers. L'apprentissage d'une langue peut se faire dans un environnement naturel ou dans un milieu scolaire. Cet apprentissage se fait d'emblée grâce à une certaine compréhension de base ainsi que par diverses interactions sociales. Cependant, certaines personnes qui ont un TDAH éprouvent des difficultés avec cette composante pragmatique, faisant en sorte que ces dernières ont des difficultés à bien communiquer dans des contextes sociaux. La pragmatique est définie comme l'usage social de la langue (Prutting et Kirchner, 1987; Searle, 1969) et est essentielle afin d'assurer le développement des autres aspects du langage (phonologie, sémantique, morphologie et syntaxe) qui sont utilisés dans des contextes conversationnels. La pragmatique est une construction hétérogène qui comprend des compétences verbales (langage) et non verbales (gestes). Les habiletés langagières générales sont typiquement corrélées avec les habiletés pragmatiques, mais les problèmes en pragmatique peuvent survenir indépendamment des problèmes de langage (Astington et Jenkins, 1999). Les troubles pragmatiques contribuent aux problèmes de performance scolaire, aux relations avec les paires et sont associés à de nombreux troubles psychiatriques (Landa, 2005). Les difficultés pragmatiques sont spécifiques à l'utilisation, telles qu'une mauvaise prise de parole, une incapacité à rester sur un sujet de conversation et une pauvre compréhension du langage dans un contexte, ce qui peut mener à une tendance à interpréter littéralement le langage figuré (Bignell et Cain, 2007).

Les problèmes sociaux apparaissent chez $52 \%$ à $82 \%$ des enfants qui ont un TDAH (Barkley, DuPaul, et McMurray, 1990; Landau, Milich, et Diener, 1998). Le rejet de ses paires est une conséquence fréquente qui empêche l'enfant d'interagir avec ceux qui l'entourent (Hoza, 2007). Les recherches qui tentent de déterminer l'étiologie du TDL chez les enfants qui ont un TDAH sont inconsistantes. Plusieurs études rapportent que les difficultés langagières chez les enfants qui ont le TDAH peuvent être principalement caractérisées par une déficience au niveau de la pragmatique (Baird, Stevenson, et Williams, 2000; Camarata et Gibson, 1999; Kim et Kaiser, 2000), tandis que d'autres ont montré que l'inattention ainsi que l'hyperactivité/impulsivité 
peuvent avoir un impact sur les aspects verbaux de la communication, tels que maintenir un sujet de conversation, ainsi que sur les aspects non verbaux comme la proximité physique appropriée (Camarata et Gibson, 1999). C'est le syndrome de la poule et de l'œuf. Est-ce le trouble du langage qui engendre des difficultés d'attention ou est-ce les difficultés d'attentions qui causent le trouble du langage ? Chez les enfants qui ont un TDAH, comme rapporté par les parents et les enseignants, les difficultés pragmatiques se caractérisent par des difficultés conversationnelles (Van Lambalgen, Van Kruistrum, et Parigger, 2008), soit un discours qui peut dévier souvent du sujet de conversation, plusieurs faux départs, des révisions et des répétitions (Redmond, 2004). Ils peuvent également présenter des problèmes au niveau des interactions avec les autres personnes. Par exemple, ils ne répondent pas ou continuent à jouer sans contact visuel, ils interrompent souvent, parlent à des étrangers de façon trop familière ou parlent de façon excessive sans prendre en considérations si quelqu'un les écoutes (Camarata et Gibson, 1999; Kim et Kaiser, 2000). De plus, ces enfants présentent des difficultés lors de l'initiation de la parole ou lors des activités qui nécessitent que l'enfant prenne des tours (Tannock, 2002). Dès la première journée ou même dans les 20 premières minutes d'interaction, ces troubles pragmatiques font en sorte que ces enfants sont moins aimés par leurs paires et qu'ils ont moins d'amis (Bickett et Milich, 1990; Erhardt et Hinshaw, 1994). Le rejet des pairs empêche l'enfant de pratiquer ses compétences sociales (Hoza, 2007). Plus tard dans leur vie, le rejet par les pairs favorise des effets néfastes, soit des problèmes scolaires ou l'abandon scolaire, la perte d'emploi, le divorce, la toxicomanie ou la délinquance (Friedman et coll., 2003; Greene, Biederman, Faraone, Sienna, et Garcia-Jetton, 1997; Klein et Mannuzza, 1991). Les enfants qui ont un TDL présentent aussi des problèmes pragmatiques. Cependant, aucune étude n'a exploré si les troubles de pragmatiques d'enfants qui ont un TDAH diffèrent des enfants qui ont un TDAH et un TDL.

\section{L'attention et l'apprentissage d'une deuxième langue}

L'apprenant d'une deuxième langue nécessite une attention particulière afin de pouvoir organiser l'information verbale qui lui arrive (Tomlin et Villa, 1994). Selon les théories de la psychologie et de la science cognitive, il n'y a aucun apprentissage sans attention (Carlson et Dulany, 1985; Fisk et Schneider, 1984; Kihlstrom, 1984; Logan, 1988; Nissen et Bullemer, 1987; Posner, 1992; Shiffrin et Schneider, 1977; Velmans, 1991; Wolford et Morrison, 1980). L'attention à l'intrant langagier est nécessaire afin que ce dernier puisse subir un traitement mental ultérieur (Carr et Curran, 1994; Scovel, 1991; Tomlin et Villa, 1994; Van Lier, 1991, 1994). De plus, les résultats des études qui ont porté sur l'attention divisée montrent que l'attention est essentielle pour l'apprentissage (Nissen et Bullemer, 1987). Aussi connue comme " multitasking », l'attention divisée comprend la concentration sur plusieurs tâches ou idées à la fois (Braun, 1998; Damos, 1991). Alors que tous les aspects notamment le vocabulaire, la syntaxe, la morphologie, la phonologie, entre autres, de l'apprentissage des langues exigent un certain degré d'attention, certains de ces aspects peuvent en nécessiter plus ou moins que d'autres (Carr et Curran, 1994; VanPatten, 1990, 1993, 1994). 


\section{Apprenant d'une L2 ayant un TDAH}

Plusieurs études ont démontré que les enfants qui ont un TDAH présentent des difficultés au niveau des compétences verbales (Andreou, Agapitou, et Karapetsas, 2005) ainsi que des difficultés avec des compétences scolaires linguistiques telles que la lecture, l'orthographe et l'écriture (Aaron, Joshi, Palmer, Smith, et Kirby, 2002; Barkley, Rischer, Edelbrock, et Smallish, 1990). Puisque certains enfants qui ont un TDAH présentent des difficultés au niveau de l'attention et de l'apprentissage de leur langue maternelle (L1), certains éducateurs ont estimé que les problèmes liés au TDAH pourraient aussi avoir un impact sur l'apprentissage d'une langue seconde (L2) (Hodge, 1998). Seulement quelques études ont examiné l'impact du TDAH sur l'apprentissage d'une L2 (Sparks, Philips, et Javorsky, 2003; Sparks, Humback, et Javorsky, 2008). Ces études ont été effectuées auprès d'étudiants du collège qui avaient un TDAH et qui étaient inscrits dans des cours d'apprentissage d'une L2. Les trois études suggèrent que les étudiants identifiés comme ayant un TDAH et inscrits à des cours de L2 n'avaient pas de difficultés en langage oral ou écrit et n'avaient pas de difficultés à réussir les cours de L2 et à satisfaire aux exigences du cours malgré leur trouble d'attention (Sparks, Philips, et Javorsky, 2003). Cependant, ces études ont seulement été effectuées auprès de jeunes adultes.

À ce jour, très peu d'études, sinon aucune n'a été effectuée sur l'impact du TDAH sur l'apprentissage d'une L2. Évaluer le langage des enfants qui ont un TDAH n'est pas une tâche facile (Cohen, Davine, Horodezky, Lipsett, et Isaacson, 1993; Little, Hart, Schatschneider, et Taylor, 2016) et comme certains étudiants qui ont un TDAH démontrent des difficultés avec l'attention ainsi que l'apprentissage de leur L1, certains chercheurs tels que Hodge (1998) soutiennent que les problèmes liés au TDAH pourraient également affecter l'apprentissage de la L2. En effet, plusieurs questions portant sur ce sujet demeurent en suspens. Les études qui ont observé l'effet du bilinguisme et du TDAH sur les fonctions exécutives montrent qu'il est toutefois possible pour cette population d'apprendre plus d'une langue avec succès (Bialystok, Hawrylewicz, Wiseheart, et Toplak, 2016; Sorge, Toplak, et Bialystok, 2017). La présente étude tentera d'explorer, pour la première fois, cette problématique. Les résultats de cette étude permettront d'acquérir des connaissances concernant les habiletés des enfants qui ont un TDAH et/ou un TDL à apprendre une L2 et, en revanche, comment les desservir plus tard afin de les appuyer. La question de recherche est la suivante : Est-ce qu'un enfant d'âge scolaire atteint d'un TDAH avec ou sans un TDL peut atteindre un niveau de compétence dans sa L2 comparable à un enfant à développement typique (DT) qui apprend une L2 dans une région où la L2 est une langue minoritaire 


\section{Aperçu de la méthode}

La présente étude est en progrès. Les chercheures évalueront environ une vingtaine d'enfants bilingues anglo-dominants de 7 à 9 ans qui ont commencé à apprendre le français après l'âge de trois ans comme L2. Parmi ces participants, ceux-ci seront divisés en trois différents groupes, soit un groupe de cinq enfants à DT, un groupe de cinq enfants qui ont un TDAH et un groupe de cinq enfants qui ont un TDAH en plus d'avoir un TDL, ces diagnostics seront confirmés par un orthophoniste et/ou un psychométricien. L'étude se ferait dans une région où le français représente la langue minoritaire et l'anglais la langue majoritaire. Des tests linguistiques en français ainsi qu'en anglais permettront d'évaluer les habiletés de ces enfants dans leurs deux langues. Des tests cognitifs seront également effectués. 


\section{Références}

Aaron, P. G., Joshi, R., Palmer, H., Smith, N., et Kirby, E. (2002). Separating genuine cases of reading disability from reading deficits caused by predominantly inattentive ADHD behavior. Journal of Learning Disabilities, 35(5), 425-435. doi:10.1177/00222194020350050301

Acheson, D. J., et Macdonald, M. C. (2009). Verbal working memory and language production: Common approaches to the serial ordering of verbal information. Psychological Bulletin, 136(3), 50-68. doi:10.1037/a0019322

Adams, C. (2008). Intervention for children with pragmatic language impairments. In C.F. Norbury, J.B. Tomblin, et D.V.M. Bishop (Eds.), Understanding developmental language disorders. From theory to practice. (pp. 189-204). New York: Psychology Press.

Alloway, T. P., Gathercole, S. E., et Elliott, J. (2010). Examining the link between working memory behaviour and academic attainment in children with ADHD. Developmental Medicine and Child Neurology, 52(7), 632-636. doi: 10.1111/j.1469-8749.2009.03603.x

Alloway, T. P., Rajendran, G., et Archibald, L. M. (2009). Working memory profiles of children with developmental disorders. Journal of Learning Difficulties, 42(4), 372-383. doi:10.1177/0022219409335214

American Psychiatric Association. (2013). Diagnostic and Statistical Manual of Mental Disorders, Fifth Edition. Washington, DC.

American Speech-Language Hearing Association. (1997). Roles of Audiologists and speechlanguage pathologists working with persons who have attention deficit hyperactivity disorder, position statement and technical report. ASHA, 39(Suppl 17), 273300.

Andreou, G., Agapitou, P., et Karapetsas, A. (2005). Verbal skills in children with ADHD. European Journal of Special Needs Education, 20(2), 231-238. doi:10.1080/08856250500055743

Åsberg, J., Kopp, S., Berg-Kelly, K., et Gillberg, C. (2010). Reading comprehension, word decoding and spelling in girls with autism spectrum disorders (ASD) and attentiondeficit/hyperactivity disorder (ADHD) : Performance and predictors. Intervention Journal of Language and Communication Disorders, 45(1), 61-71. doi:10.3109/13682820902745438

Astington, J. W., et Jenkins, J. M. (1999). A longitudinal study of the relation between language and theory-of-mind development. Developmental. Psychology, 35(5), 1311-1320. doi:10.1037/0012-1649.35.5.1311

Baird, J., Stevenson, J. C., et Williams, D. C. (2000). The evolution of ADHD: a disorder of communication?. The Quarterly Review of Biology, 75(1), 17-35. Doi:10.1086/393256 
Barkley, R. A. (1994). Impaired delayed responding: A unified theory of attention deficit hyperactivity disorder. In D. K. Routh (Ed.), Disruptive behavior disorders: Essays in honor of Herbert Quay (pp. 11-57). New York: Plenum

Barkley, R. A. (1997). ADHD and the Nature of Self-control (New York, NY: Guilford).

Barkley, R. A. (2006). Attention-deficit hyperactivity disorder: A handbook for diagnosis and treatment. $3^{\text {rd }}$ ed. Guilford Press; New York

Barkley, R. A., DuPaul, G. J., et McMurray, M. B. (1990). Comprehensive evaluation of attention deficit disorder with and without hyperactivity as defined by research criteria. Journal of Consulting and Clinical Psychology, 58(6), 775-789. doi: 10.1037/0022006X.58.6.775

Barkley, R., Rischer, M., Edelbrock, C., et Smallish, L. (1990). The adolescent outcome of hyperactive children diagnosed by research criteria: An 8-year prospective follow-up study. Journal of the American Academy of Child and Adolescent Psychiatry, 29(4), 546557. doi: 10.1097/00004583-199007000-00007

Benton, A. (1991). Prefrontal injury and behavior in children. Developmental Neuropsychology, 7(3), 275-281. Doi: 10.1080/87565649109540495

Bialystok, E., Hawrylewics, K., et Wiseheart, M. (2016). Interaction of bilingualism and attention-deficit/hyperactivity disorder in young adults. Bilingualism: Language and Cognition, 20(3), 588-601. doi:10.1017/S1366728915000887

Bickett, L., et Milich, R. (1990). First impressions formed of boys with learning disabilities and attention deficit disorder. Journal of learning disabilities, 23(4), 253-259. Doi:

10.1177/002221949002300409

Bignell, S., et Cain, K. (2007). Pragmatic aspects of communication and language comprehension in groups of children differentiated by teacher ratings of inattention and hyperactivity. British Journal of Developmental Psychology, 25(4), 499512doi:10.1348/026151006X171343

Braun, J. (1998). Divided attention: narrowing the gap between brain and behavior. In R.

Parasuraman (Ed.), The attentive brain (pp.327-351). Cambridge, MA: MIT Press.

Brossard-Racine, M., Shevell, M., Snider, L., Ageranioti Bélanger, S., Julien, M., et Majnemer, A. (2015). Persistent handwriting difficulties in children with ADHD after treatment with stimulant medication. Journal of Attention Disorders, 19(7), 620-629.

doi:10.1177/1087054712461936

Bruce, B., Thernlund, G., et Nettelbladt, U. (2006). ADHD and language impairment. A study of parent questionnaire FTF (Five to Fifteen). European Child and Adolescent Psychiatry, 15(1), 52-60. doi :10.1007/s00787-006-0508-9 
Camarata, S., et Gibson, T. (1999). Pragmatic language deficits in attention-deficit hyperactivity disorder (ADHD). Developmental Disabilities Research Reviews, 5(3), 207-214. doi:10.1002/(SICI)1098-2779(1999)5:3<207::AID-MRDD7>3.0.CO;2-O

Cantwell, P. D. (1996). Attention deficit disorder: a review of the past 10 years. Journal of the American Academy of Child and Adolescent Psychiatry, 35(8), 978-987. doi:10.1097/00004583-199608000-00008

Carlson, R. A., et Dulany, D. E. (1985). Conscious attention and abstraction in concept learning. Journal of Experimental Psychology: Learning, Memory, and Cognition, 11(1), 45-58. doi:10.1037/0278-7393.11.1.45

Carr, T. H., et Curran, T. (1994). Cognitive factors in learning about structured sequences: Applications to syntax. Studies in Second Language Acquisition, 16(2), 205-230. doi :10.1017/S0272263100012882

Cepeda, N. J., Cepeda, M. L., et Kramer, A. F. (2000). Task switching and Attention Deficit Hyperactivity Disorder. Journal of Abnormal Child Psychology, 28(3), 213-226. doi:10.1023/A:1005143419092

Canadian Mental Health Association (CMHA). (2012). Attention Deficit Disorders. Accédé Sept 15. http://www.cmha.ca/mental-health/understanding-mental-illness/attentiondeficitdisorders/

Cohen, N. J., Davine, M., Horodezky, N., Lipsett, L., et Isaacson, L. (1993). Unsuspected language impairment in psychiatrically disturbed children: Prevalence and language and behavioral characteristics. Journal of the American Academy of Child and Adolescent Psychiatry, 32(3), 595-603. doi: 10.1097/00004583-199305000-00016

Cohen, N. J., Vallance, D. D., et Barwick, M. (2000). The interface between ADHD and language impairment : An examination of language, achievement, and cognitive processing. Journal of Child Psychology and Psychiatry and Allied Disciplines, 41(3), 353-362. Repéré sur: https://www.cambridge.org/core/journals/journal-ofchildpsychology-and-psychiatry-and-allied-disciplines/article/the-interface-betweenadhd-andlanguage-impairment-an-examination-of-language-achievement-andcognitiveprocessing/46161F5E20CEDCF91B61A7F81E270735

Cross, M. (2011). Children with social, emotional, and behavioural difficulties and communication problems. There is always a reason. London: Jessica Kingsley Publishers

Damos, D. (1991). Dual-task methodology: Some common problems. In D. L. Damos (Ed.), Multiple-task performance (pp. 101-119). Washington, DC: Taylor et Francis.

Diamond, A. (2014). Executive functions. Annual Review of Psychology, 64(1), 135-168. doi:10.1146/annurev-psych-113011-143750 
Douglas, V. (1988). Cognitive deficits in children with attention deficit disorder with hyperactivity. In L. M. Bloomingdale et J. A. Sergeant (Eds.), Attention deficit disorder: Criteria, cognition, intervention (pp. 65-82). London: Pergamon.

Douglas, V. (2005). Cognitive deficits in children with attention deficit hyperactivity disorder: A long-term follow-up. Canadian Psychology, 46(1), 23-31. doi:10.1037/h0085821

Erhardt, D., et Hinshaw, S. P. (1994). Initial sociometric impressions of attention-deficit hyperactivity disorder and comparison boys: predictions from social behaviors and from nonbehavioral variables. Journal of Consultation Clinic Psychology, 62(4), 833-842. doi:10.1037/0022-006X.62.4.833

Ervin, M. (2001). SLI: what we know and why it matters. The ASHA Leader, 6(1), 4-31. doi: 10.1044/leader.FTR1.06122001.4

Fierro-Cobas, V. et Chan, E. (2001). Language development in bilingual children: A primer for pediatricians. Contemporary Pediatrics, 18(7), 79-98. Repéré sur:http://courses.washington.edu/sop/Bilingualism_PrimerPediatricians.pdf

Fisk, A. D., et Schneider, W. (1984). Memory as a function of attention, level of processing, and automatization. Journal of Experimental Psychology: Learning, Memory, and Cognition, 10(2), 181-197. doi:10.1037/0278-7393.10.2.181

Friedman, S. R., Rapport, L. J., Lumley, M., Tzeleppis, A., VanVoorhis, A., Stettner, L., et Kakaati, L. (2003). Aspects of social and emotional competence in adult-attention-deficit hyperactivity disorder. Neuropsychology, 17(1), 50-58. doi:10.1037/0894-4105.17.1.50

Gathercole, S. E., et Baddeley, A. D. (1990). Phonological memory deficits in language disordered children: Is there a causal connection?. Journal of Memory and Language, 29(3), 336-360. doi:10.1016/0749-596X(90)90004-J

Gathercole, S.E., Brown, L., et Pickering, S.J. (2003). Working memory assessments at school entry as longitudinal pre- dictors of national curriculum attainment levels. Educational and Child Psychology, 20(3), 109-122. Repéré

sur:https://www.researchgate.net/profile/Lorna_Bourke/publication/250928054_The_rela tionship_between_working_memory_and_early_writing_at_the_word_sentence_and_text _level/links/02e7e51ee5262398b0000000.pdf\#page=111

Gathercole, S. E., Pickering, S. J., Knight, C., et Stegmann, Z. (2004). Working memory skills and educational attainment: Evidence from national curriculum assessments at 7 and 14 years of age. Applied Cognitive Psychology, 18(1), 1-16. doi: 10.1002/scp.934

Geurts, H. M., Broeders, M., et Nieuwland, M. S. (2010). Thinking outside the executive functions box: theory of mind and pragmatic abilities in attention deficit/hyperactivity dis- order. European Journal of Developmental Psychology, 7(1), 135- 151. doi:10.1080/17405620902906965 
Greene, R. W., Biederman, J., Faraone, S. V., Sienna, M., et Garcia-Jetton, J. (1997). Adolescent outcome of boys with attention-deficit/hyperactivity disorder and social disability : Results from a 4-year longitudinal follow-up study. Journal Consultation Clinic of Psychology, 65(5), 758-767. doi :10.1037/0022-006X.65.5.758

Gropper, R. J., et Tannock, R. A. (2009). A pilot study of working memory and academic achievement in college students in ADHD. Journal of Attention Disorders, 12(6), 574581. doi:10.1177/1087054708320390

Guldberg-Kjär, T., et Johansson, B. (2015). ADHD symptoms across the lifespan: A comparison of symptoms captured by the wender and barkley scales and DSM-IV criteria in a population-based swedish sample aged 65 to 80. Journal of Attention Disorders, 19(5), 390- 404. doi:10.1177/1087054713514853

Hakansson, G., et Nettelbladt, U. (1993). Developmental sequences in L1 (normal and impaired) and L2 acquisition of Swedish syntax. International Journal of Applied Linguistics, 3(1), 3-29. doi: 10.1111/j.1473-4192.1993.tb00047.x

Helland, W. A., Biringer, E., Helland, T., et Heimann, M. (2010). Exploring language profiles for children with ADHD and children with asperger syndrome. Journal of Attention Disorders, 16(1), 34-43. doi:10.1177/1087054710378233

Hervey, A. S., Epstein, J. N., et Curry, J. F. (2004). Neuropsychology of adults with AttentionDeficit Hyperactivity Disorder: A meta-analytic review. Neuropsychology, 18(3), 485503. doi:10.1037/0894-4105.18.3.485

Hodge, M. E. (1998). Teaching foreign language to at-risk learners: A challenge for the new millennium. Inquiry, 2(1), 68-78. Repéré sur: https://eric.ed.gov/?id=EJ591728

Hoza, B. (2007). Peer functioning in children with ADHD. Journal of Pediatric Psychology, 32(6), 655-663. doi:10.1093/jpepsy/jsm024

Hulme, C., et Snowling, M. J. (2009). Developmental Disorders of Language Learning and Cognition. Singapore: Wiley- Blackwell.

Jonsdottir, S., Bouma, A., Sergeant, J. A., et Scherder, E. J. A. (2005). The impact of specific language impairment on working memory in children with ADHD combined subtype.

Archives of Clinical Neuropsychology, 20(4), 443-456. doi:10.1016/j.acn.2004.10.004

Kihlstrom, J. (1984). Conscious, subconscious, unconscious: A cognitive perspective. In K. Bowers et D. Meichenbaum (Eds.), The unsconscious reconsidered (pp. 149-211). NewYork: Wiley

Kim, O., et Kaiser, A. (2000). Language characteristics of children with ADHD. Commununication Disorders Quarterly, 21(3), 154-165. doi :10.1177/152574010002100304 
King, J. A., Colla, M., Brass, M., Heuser, I., et Von Cramon, D. Y. (2007). Inefficient cognitive control in adult ADHD: Evidence from trial-by-trial stroop tests and cued task switching performance. Behavioral and Brain Functions, 3(42). doi:10.1186/1744-9081-3-42

Klein, R. G., et Mannuza, S. (1991). Long-term outcome of hyperactive children: a review. Journal of the American Academy of Child and Adolescent Psychiatry, 30(3), 383-387. doi:10.1097/00004583-199105000-00005

Kovacs A. M., et Mehler, J. (2009). Cognitive gains in 7-month-old bilingual infants. Proceedings of the National Academy of Science, 106(16), 6556-6560. doi:10.1073/pmsa.0811323106.

Kramer, A. F., Cepeda, N. J., et Cepeda, C. L. (2001). Methylpheniadate effects on taskswitching performance in attention-deficit/hyperactivity disorder. Journal of the American Academy of Child and Adolescent Psychiatry, 40(11), 1277-1284. doi:10.1097/00004583200111000-00007

LaForette, D. R., Murray, D. W., et Kollins, S. H. (2008). Psychosocial treatments for preschoolaged children with attention -deficit hyperactivity disorder. Developmental Disabilities Research Reviews, 14(1), 300-310. doi:10.1002/ddrr.36

Landa, R. J. (2005). Assessment of social communication skills in preschoolers. Mental Developmental Disabilities Reviews, 11(3), 247-252. doi :10.1002/mrdd.20079

Landau, S., Milich, R., et Diener, M. (1998). Peer relations of children with attention-deficit hyperactivity disorder. Reading and Writing Quarterly: Overcoming Learning Difficulties, 14(1), 83-105. doi:10.1080/1057356980140105

Leonard, L. B. (1998). Children with specific language impairment. Cambridge, MA: MIT Press.

Little, C. W., Hart, S. A., et Schatschneider, C. (2014). Examining associations among ADHD, homework behavior, and reading comprehension. Journal of Learning Disabilities, 49(4), 410-423. doi:10.1177/0022219414555715

Logan, G. D. (1988). Toward an instance theory of automatization. Psychological Review, 95(4), 492-527. doi:10.1037/0033-295X.95.4.492

Love, A. J., et Thompson, M. G. G. (1988). Language disorders and attention deficit disorders in young children referred for psychiatric services: analysis of prevalence and a conceptual synthesis. American Journal of Orthopsychiatry, 58(1), 52-64.

doi:10.1111/j.19390025.1988.tb01566.x

Lui, M., et Tannock, R. (2007). Working memory and inattentive behaviour in a community sample of children. Behavioral and Brain Functions, 3(12). doi:10.1186/1744-9081-3-12

Martinussen, R., Hayden, J., Hogg-Johnson, S., et Tannock, R. (2005). A meta-analysis of working memory impairments in children with attention-deficit/hyperactivity disorder. 
Journal of the American Academy of Child and Adolescent Psychiatry, 44(4), 377-384. doi:10.1097/01.chi.0000153228.72591.73

Mathers, M. E. (2006). Aspects of language in children with ADHD. Journal of Attention Disorders, 9(3), 523-533. doi:10.1177/1087054705282437

Mattes, J. A. (1980). The role of frontal lobe dysfunction in childhood hyperkinesis. Comprehensive Psychiatry, 21(5), 358-369. doi:10.1016/0010-440X(80)90017-6

Mayes, S. D., Calhoun, S. L., et Crowell, E. W. (2000). Learning disabilities and ADHD. Overlapping Spectrum Disorders, 33(5), 417-424. doi:10.1177/002221940003300502

Mayes, R., et Rafalovich, A. (2007). Suffer the restless children: The evolution of ADHD and paediatric stimulant use, 1900-80. History of Psychiatry, 18(4), 435-457. doi:10.1177/0957154X06075782

McInnes, A., Humphries, T., Hogg-Johnson, S., et Tannock, R. (2003). Listening comprehension and working memory are impaired in attention-deficit-hyperactivity disorder irrespective of language impairment. Journal of Abnormal Child Psychology, 31(4), 427-443. doi:10.1023\%2FA\%3A1023895602957

McGrath, L. M., Pennington, B. F., Shanahan, M. A., Santerre-Lem- mon, L. E., Barnard, H. D., Willcut, E. G., et Olson, R. K. (2011). A multiple deficit model of reading disability and attention-deficit/ hyperactivity disorder: Searching for shared cognitive deficits. Journal of Child Psychology and Psychiatry, 52(1), 547-557. doi:

10.1111/j.14697610.2010.02346.x

NIH Consensus Development Panel. (2000). National Institutes of Health Consensus Development Conference statement: Diagnosis and treatment of attentiondeficit/hyperactivity disorder (ADHD). Journal of the American Academy of Child and Adolescent Psychiatry, 39(2), 182-193. doi: 10.1097/00004583-200002000-00018

Nigg, J. T. (2005). Neuropsychologic theory and findings in attention-deficit/hyperactivity disorder: The state of the field and salient challenges for the coming decade. Biological Psychiatry, 57(11), 1424-1435. doi:10.1016/j.biopsych.2004.11.011

Nissen, M., et Bullemer, P. (1987). Attentional requirements of learning: Evidence from performance measures. Cognitive Psychology, 19(1), 1-32. doi:10.1016/00100285(87)90002-8

Norbury, C. F., Gooch, D., Wray, C., Baird, G., Charman, T., Simonoff, E., et Pickles, A. (2016). The impact of nonverbal ability on prevalence and clinical presentation of language disorder: Evidence from a population study. Journal of Child Psychology and Psychiatry, 57(4), 1247-1257. doi:10.1111/jcpp.12573

Özerk, M. R., Handorff, J. A., et Özerk, K. (2011). Assessment of bilingual children with inattention, over activity and impulsivity - Challenges and solutions. International Electronic Journal of Elementary Education, 3(3), 193-212. Repéré 
sur:https://search.proquest.com/openview/8dd7f1dc9152661a63cdc81dc56f2949/1?pqori gsite $=$ gscholar \&cbl $=656305$

Papaeliou, C. F., Maniadaki, K., et Kakouros, E. (2015). Association between story recall and other language abilities in schoolchildren with ADHD. Journal of Attention Disorders, 19(1), 53- 62. doi:10.1177/1087054712446812

Paradis, J. (2005). Grammatical morphology in children learning English as a second language: Implications of similarities with Specific Language Impairment. Language, Speech and

Hearing Services in the Schools, 36(1), 172-187. doi:10.1044/0161-1461(2005/019) Paradis, J. (2011). Individual differences in child English second language acquisition: Comparing child-internal and child-external factors. Linguistic Approaches to Bilingualism, 1(3), 213-237. doi:10.1075/lab.1.3.01par

Paradis, J. et Crago, M. (2000). Tense and temporality: Similarities and differences between language- impaired and second-language children. Journal of Speech, Language and Hearing Research, 43(1), 834-848. doi:10.1044/jslhr.4304.83

Pennington, B. E, Grossier, D., et Welsh, M.C. (1993). Contrasting cognitive deficits in attention deficit disorder versus reading disability. Developmental Psychology, 29(3), 511-523. doi:10.1037/0012-1649.29.3.511

Posner, M. I. (1992). Attention as a cognitive and neural system. Current Directions in Psychological Science, 1(1), 11-14. doi: 10.1111/1467-8721.ep10767759

Prutting, C. A., et Kirchner, D. M. (1987). A clinical appraisal of the pragmatic aspects of language. Journal of Speech et Hearing Disorders, 52(2), 105-119. Repéré sur:https://www.lamar.edu/fine-artscommunication/_files/documents/speechhearing/prutting_checklist_article.pdf

Purvis, K. L., et Tannock, R. (1997). Language abilities in children with attention deficit hyperactivity disorder, reading disabilities, and normal controls. Journal of Abnormal Child Psychology, 25(2), 133-144. doi:10.1023\%2FA\%3A1025731529006

Redmond, S. (2004). Conversational profiles of children with ADHD, SLI, and typical development. Clinical Linguistics and Phonetics, 18(2), 107-125. doi:10.1080/02699200310001611612

Redmond, S. M., Thompson, H. L., et Goldstein, S. (2011). Psycholinguistic profiling differentiates specific language impairment from typical development and from attentiondeficit /hyperactivity disorder. Journal of Speech, Language and Hearing Research, 54(1), 99-117. doi:10.1044/1092-4388(2010/10-0010)

Rice, M. L. (2004). Growth models of developmental language disorders. In M. L. Rice et S. F. Warren (Eds.), Developmental language disorders: From phenotypes to etiologies (p. 207-240). Mahwah, NJ: Erlbaum. 
Rogers, M., Hwang, H., Toplak, M., Weiss, M., et Tannock, R. (2011). Inattention, working memory, and academic achievement in adolescents referred for attention deficit/hyperactivity disorder (ADHD). Child Neuropsychology, 17(5), 444-458. doi:10.1080/09297049.2010.544648

Rutter, M., Silberg, J., O’Connor, T., et Simonoff, E. (1999). Genetics and child psychiatry: empirical research findings. Journal of Child Psychology and Psychiatry, 40(1), 19-55. Repéré sur:https://www.cambridge.org/core/journals/journal-of-child-psychologyandpsychiatry-and-allied-disciplines/article/genetics-and-child-psychiatry-iiempiricalresearch-findings/DE7B4BCBB4C21C4E1B760944E8061753

Scahill, L. et Schwab Stone, M. (2000). Epidemiology of ADHD in school-age children. Child and Adolescent Psychiatric Clinics of North America, 9(3), 541-555. Repéré sur:http://psycnet.apa.org/record/2000-00431-006

Schachar, R. J., Tannock, R., et Logan, G. (1993). Inhibitory control, impulsiveness, and attention deficit hyperactivity disorder. Clinical Psychology Review, 13(8), 721-739. doi: 10.1016/S0272-7358(05)80003-0

Scovel, T. (1991). Attention, apperception, awareness, and acquisition. Paper presented at the Thai TESOL Eleventh Annual Convention, Bangkok

Searle, J. R. (1969). Speech acts. Cambridge: Cambridge University Press.

Singh, L., Fu C. S., Rahman, A. A., Hameed, W. B., Sanmugam, S., Agarwal, P., Jiang, B., Chong, Y. S., Meaney, M. J., Rifkin-Graboi, A. (2014). Back to basics: A bilingual advantage in infant visual habituation. Child Development, 86(1), 294-302. doi:10.1111/cdev.12271.

Shiffrin, R. M., et Schneider, W. (1977). Controlled and automatic human information processing II : Perceptual learning, automatic attending, and a general theory. Psychological Review, 84(2), 127-190. doi:10.1037/0033-295X.84.2.127

Smalley, S. L. (1997). Genetic influences in childhood-onset psychiatric disorders: autism and attention deficit/hyperactivity disorder. American Journal of Human Genetics, 60(6), 1276-1282. doi:10.1086/515485

Snowling, M. J., Bishop, D. V. M., Stothard, S. E., Chipchase, B., et Kaplan, C. (2006). Psychosocial outcomes at 15 years of children with a preschool history of speechlanguage impairment. Journal of Child Psychology and Psychiatry, 47(8), 759-765. doi:10.1111/j.1469-7610.2006.01631.x

Sonuga-Barke, E. J. (2003). The dual pathway model of ADHD: an elaboration of neurodevelopmental characteristics. Neuroscience et Behavioral Reviews, 27(7), 593604. doi:10.1016/j.neubiorev.2003.08.005

Sonuga-Barke, E. J., Bitsakou, P., et Thompson, M. (2010). Beyond the dual pathway model: Evidence for the dissociation of timing, inhibitory, and delay-related impairments in 
attention-deficit/hyperactivity disorder. Journal of American Academy of Child and Adolescent Psychiatry, 49(4), 345-355. doi:10.1016/j.jaac.2009.12.018

Sorge, G. B., Toplak, M. E., et Bialystok, E. (2017). Interactions between levels of attention ability and levels of bilingualism in chilren's executive functioning. Developmental Science, 20(1), 130-141. doi:10.1111/desc.12408

Sparks, R., Humback, N., et Javorsky, J. (2008). Individual and longitudinal differences among high and low-achieving, LD, and ADHD L2 learners. Learning and Individual Differences, 18(1), 29-43. doi:10.1016/j.lindif.2007.07.003

Sparks, R., Philips, L., et Javorsky, J. (2003). College students classified as having learning disabilities and attention deficit hyperactivity disorder and the foreign language requirement. Foreign Language Annals, 36(3), 325-338. doi:10.1111/j.19449720.2003.tb02117.x

Swanson, L. (2006). Cognitive processes that underlie mathematical precociousness in young children. Journal of Experimental Child Psychology, 93(2), 239-264. doi:10.1016/j.jecp.2005.09.006

Tannock R (2002) Language, Reading, and Motor Control Problems in ADHD. A potential Behavioral Phenotype. In: Greenhill LL (ed) Learning Disabilities, Implications for

Psychiatric Treatment. Washington, DC, London, England, American Psychiatric Press Inc

Tannock, R., et Schachar, R. (1996). Executive dysfunction as an underlying mechanism of behavior and language problems in attention deficit hyperactivity disorder. In J. H. Beitchman, N. J. Cohen, M. M. Konstantareas and R. Tannock (Eds) Language, Learning, and Behaviour Disorders: Developmental, biological, and clinical perspectives (Cambridge: Cambridge University Press), pp. 128-155

Tirosh, E., et Cohen, A. (1998). Language deficit with attention-hyperactivity disorder: a prevalent comorbidity. Journal of Child Neurology, 13(10), 493-497. doi:10.1177/088307389801301005

Tomlin, R. S., et Villa, V. (1994). Attention in cognitive science and second language acquisition. Studies in Second Language Acquisition. 16(2), 183-203. doi:10.1017/S0272263100012870

Toplak, M. E., Rucklidge, J. J., Hetherington, R., John, S. C. F., et Tannock, R. (2003). Time perception deficits in attention-deficit/ hyperactivity disorder and comorbid reading difficulties in child and adolescent samples. Journal of Child Psychology and Psychiatry, 4(6), 888-903. doi:10.1111/1469-7610.00173

Van Lambalgen, M., Val Kruistum, C., et Parigger, E. (2008). Discourse processing in attentiondeficit hyperactivity disorder (ADHD). Journal of Logic Language and Information. 17(2), 467-487. doi: 10.1007\%2Fs10849-008-9066-5 
Van Lier, L. (1991). Inside the classroom: Learning processes and teaching procedures. Applied Language Learning, 2(1), 29-68. Repéré sur:https://eric.ed.gov/?id=EJ430050

Van Lier, L. (1994). Language awareness, contingency, and interaction. AILA Review, 11(1), 6982. Repéré sur:https://s3.amazonaws.com/academia.edu.documents/42919193/AILA11.pdf?AWSAc cessKeyId=AKIAIWOWYYGZ2Y53UL3A\&Expires $=1513453446 \&$ Signature $=\mathrm{gbsKpPa}$ C6z2OrEC\%2BJ164\%2FkCNNJI\%3D\&responsecontentdisposition=inline $\% 3 \mathrm{~B} \% 20$ filename $\% 3$ DAILA11.pdf\#page $=69$

VanPatten, B. (1990). Attending to content and form in the input: An experiment in consciousness. Studies in Second Language Acquisition, 12(3), 287-301. doi :https://doi.org/10.1017/S0272263100009177

VanPatten, B. (1993). Grammar teaching for the acquisition-rich classroom. Foreign Language Annals, 26(4), 435-450. doi:10.1111/j.1944-9720.1993.tb01179.x

VanPatten, B. (1994). Evaluating the role of consciousness in second language acquisition: Terms, linguistic features et research methodology. AILA Review, 11(1), 27-36. Repéré sur:https://www.researchgate.net/profile/Rick_Graaff/publication/254771816_Under_wh at_conditions_does_explicit_knowledge_of_a_second_language_facilitate_the_acquisitio n_of_implicit_knowledge_A_research_proposal/links/53e8bcb20cf25d674ea8590a.pdf\#p age $=27$

Velmans, M. (1991). Is human information processing conscious?. Behavioral and Brain Sciences, 14(4), 651-669. doi:https://doi.org/10.1017/S0140525X00071776

Westby, C. E., et Cutler, S. K. (1994). Language and ADHD: understanding the bases and treatment of self-regulatory deficits. Topics in Language Disorders, 14(1), 58-76. Repéré sur:http://journals.lww.com/topicsinlanguagedisorders/Abstract/1994/08000/Language_a nd_ADHD_Understanding_the_bases_and.6.aspx

Whitehouse, A. J. O., Line, E. A., Watt, H. J., et Bishop, D. V. M. (2009). Qualitative aspects of developmental language impairment relate to language and literacy outcome in adulthood. International Journal of Language and Communication Disorders, 44(4), 489-509. doi:10.1080/13682820802708080

Wolford, G., et Morrison, F. (1980). Processing of unattended visual information. Memory et Cognition, 8(6), 521-527. doi:10.3758\%2FBF03213771 